Results Two patients with PDOC were admitted to the hospice over a 12 month period for withdrawal of CANH. A 28-yearold man who had been in a vegetative state for 15 months secondary to a cerebral abscess and a 73-year-old woman who had been in a vegetative state for 6 years following a hypoxic brain injury. They both died at the hospice 9 days after discontinuing CANH. Key themes identified were educational needs of hospice staff especially an understanding of how reflex reactions may be misunderstood as purposeful actions in PDOC, the value of providing an opportunity for staff to debrief following episodes of care and the importance of the hospice team being involved in the development of a detailed end of life plan prior to hospice admission.

Conclusion There are numerous medical, ethical and legal challenges encountered in making the decision to withdraw $\mathrm{CANH}$ and then in providing end of life care for these patients. Learning from our work may help other professionals caring for similar patients as we predict an increase in referrals to hospices to be involved in managing the care of similar patients.

\section{A CASENOTE REVIEW OF PARENTERAL INFUSIONS IN DYING PATIENTS; A RESPONSE TO RECENT MEDIA HYPE}

Eilidh Burns, Carol Davis. NHS University Hospital Southampton Foundation Trust

\subsection{6/bmjspcare-2019-ASP.55}

Background In the aftermath of the Gosport Independent Panel Report (June 2018), the National Audit of Care at End of Life presented an opportunity to examine the use of continuous parenteral infusions for symptom relief in dying patients. Methods Casenote review of acute hospital in-patients who died in April 2018 recording patient location, diagnosis and hospital palliative care team (HPCT) involvement. For patients who died on an infusion MDT involvement, rationale for starting infusion, duration and drugs used were recorded.

Results 86 casenotes reviewed. 34/86 (40\%) patients died on an infusion.

$21 / 60(33 \%)$ dying of non-malignant conditions and 13/26 $(50 \%)$ dying of cancer were on an infusion. 29/49 (59\%) being reviewed by the HPCT and $5 / 37$ (15\%) not known to HPCT were prescribed an infusion. 9/14 (64\%) patients on an oncology ward and $8 / 28(29 \%)$ on a care of the elderly (COTE) ward were on infusions.

$30 / 34(88 \%)$ started infusions including an opioid (12 morphine, range $5-30 \mathrm{mg}$, median $10 \mathrm{mg} ; 18$ oxycodone, range 5-20 mg, median $5 \mathrm{mg}$ ). 33/34 (97\%) died with an opioid (12 morphine, range 5-30, median $10 \mathrm{mg} ; 21$ oxycodone, range $5-50 \mathrm{mg}$, median $5 \mathrm{mg}$ ). 19/21 (90\%) receiving infusional oxycodone were known to HPCT. Other drugs infused: midazolam (21), anti-secretory (18), levomepromazine (10), haloperidol (5), metoclopramide (2).

Mean infusion duration 2.2 days (range $0-13$ ).

Conclusions Patients with non-malignant conditions and those on COTE wards were less likely to die on an infusion. Not all patients with cancer, nor all those known to HPCT, died on an infusion, however, patients on an infusion were more likely to be known to HPCT.

Average doses of opioids on starting were low and did not substantially increase, which could be related to the short duration of infusions. Poor documentation precluded detailed qualitative analysis.

\section{INEQUALITIES IN HOSPICE CARE PROVISION: A SYSTEMATIC LITERATURE REVIEW AND NARRATIVE SYNTHESIS}

Alice Rogers, Jake Tobin, Sebastian Tullie, Asanish Kalyanasundaram, Isla Kuhn, Stephen Barclay. University of Cambridge School of Clinical Medicine

\subsection{6/bmjspcare-2019-ASP.56}

Background Hospice care is often perceived as being associated with stereotypical patients: white, middle-class, young and with cancer.

Aim To review the literature concerning who is/is not referred for hospice care, with a focus on diagnosis, age, gender, marital status, ethnicity, geography and socio-economic status.

Design of study Systematic literature review and narrative synthesis.

Methods Searches of Medline, PsycINFO, CINAHL, Web of Science, Assia and Embase databases from January 1987 to December 2017 were conducted, with further citation, reference and hand searches. Studies of adult patients living in the UK/Australia/New Zealand and Canada and receiving a range of hospice care (inpatient, outpatient, day hospice, hospice at home, community specialist (Macmillan) nursing) were included. Case studies, opinion pieces and systematic reviews were excluded. Of 28433 articles reviewed, 152 met the inclusion criteria. Extracted data were analysed using NVivo with a narrative synthesis of emergent themes.

Results Since 1987, there has been extensive research demonstrating inequalities in hospice care provision. There is some evidence that some inequalities are reducing, particularly for those with non-malignant diseases. Nonetheless, there are still important groups with less access to hospice care, including the oldest old, ethnic minorities and those living in areas of high socioeconomic deprivation. Gender and marital status have little effect on hospice care provision. The literature concerning hospice care provision for the LGBTQ community and those living with HIV/AIDs is very limited.

Conclusion Prognostic uncertainty and fragmentation of care remain barriers to receipt of hospice care for those with nonmalignant disease. The oldest-old, the socio-economically deprived and those from ethnic minorities continue to receive less hospice care. There is a persisting challenge to the hospice movement to provide equitable access to all.

\section{THE DEVELOPMENT OF A FRAMEWORK TO PERSONALISE HYDRATION MANAGEMENT IN CANCER CARE: THE USE OF NON-INVASIVE TECHNOLOGY TO EVALUATE FLUID STATUS AND DEHYDRATION-RELATED SYMPTOMS}

Amara Callistus Nwosu, Sarah Stanley, Alexandra McDougall, Catriona R Mayland, Stephen Mason, Fran Westwell, Joanne Bell, Trevor F Cox, Andrea Varro, John E Ellershaw. Palliative Care Institute Liverpool (PCIL) - University of Liverpool, Royal Liverpool and Broadgreen University Hospitals NHS Trust, Marie Curie Hospice Liverpool, Liverpool Cancer Trials Unit, School of Physiological Sciences - University of Liverpool

\subsection{6/bmjspcare-2019-ASP.57}

Background The role of hydration in causing or alleviating suffering in advanced cancer is poorly understood. The evidence for the efficacy of clinically assisted hydration in advanced cancer is inconclusive. Bioelectrical impedance vector analysis (BIVA) is an accurate validated method of assessing hydration status. Previous work with BIVA demonstrates 